\title{
A Rare Case of a Giant Adrenal Incidentaloma in 78 Year Old: Incidence, Radiologic and Management Issues of Adrenal Myelolipoma
}

\author{
EddSherwyn Luke Q Tan, and Gerry H Tan*
}

Department of Medicine, Cebu Doctors University Hospital, Philippines

*Corresponding author: Gerry H Tan, Division of Endocrinology, Diabetes and Metabolism, Cebu Doctors University College of Medicine, Cebu Doctors University Hospital, Philippines, E-mail: endoking2@yahoo.com.ph

Received: 11 Nov, 2019 | Accepted: 09 Dec, 2019 | Published: 16 Dec, 2019

Citation: Tan ELQ, Tan GH (2019) A Rare Case of a Giant Adrenal Incidentaloma in 78 Year Old: Incidence, Radiologic and Management Issues of Adrenal Myelolipoma. Int J Endocrinol Metab Disord 5(3): dx.doi.org/10.16966/2380-548X.162

Copyright: (C) 2019 Tan ELQ, et al. This is an open-access article distributed under the terms of the Creative Commons Attribution License, which permits unrestricted use, distribution, and reproduction in any medium, provided the original author and source are credited.

\section{Abstract}

Adrenal myelolipoma is a rare benign neoplasm composed of mature adipose and hematopoietic tissue. Most lesions are small, unilateral and asymptomatic, discovered incidentally at autopsy or imaging studies performed for other reasons [1]. The incidence of Adrenal incidentalomas is at least $2 \%$ in the general population [2]. The prevalence of finding an adrenal myelolipoma from an incidentaloma is very small. In autopsy studies, the incidence of adrenal myelolipoma is reported as being 0.08 to $0.4 \% 1$ with a male-to-female ratio of $1: 1$. No other documented case of Adrenal Myelolipoma was ever reported in our institution and only 1 published case of Adrenal Myelolipoma locally [3].

The present case is a 78-year old Filipino man who on executive check-up had an incidental finding of adrenal mass. Biochemical evaluation revealed that this was a non- functioning adrenal mass. Further evaluation including MRI of the adrenal gland showed a right suprarenal mass lesion with enhancing solid and fat components consistent with Myelolipoma.

Since population based studies have limited data regarding the epidemiology of Adrenal myelolipoma, we are reporting this case to help future research studies.

\section{Introduction}

Adrenal myelolipoma is a rare benign neoplasm composed of mature adipose and hematopoietic tissue. Most lesions are small, unilateral and asymptomatic, discovered incidentally at autopsy or on imaging studies performed for other reasons [1]. They cause no adrenal dysfunction or hematologic disorder [2]. It was initially described by Gierke in 1905 and subsequently termed as formations myelolipomatoses by Oberling in 1929. The tumor appears to affect men and women equally and most commonly found between the fifth and the seventh decade of life [4]. Accounting for 3-5\% of all primary tumors of the adrenals, the true incidence of these tumors is not known, although it is thought to be $0.08 \%-0.4 \%$, with increased incidence noted in the later decades of life [1]. They are generally nonsecreting in nature, and only one case of secreting myelolipoma has been reported so far [3].

\section{Case Presentation}

This is a case of a 78-year-old Filipino male, hypertensive (controlled with Irbesartan $150 \mathrm{mg}$ daily), prediabetic, non-asthmatic, a 15-pack year smoker, and occasional alcoholic beverage drinker. On routine evaluation, ultrasound of the whole abdomen revealed a suspicious mass proximate to the liver. Further imaging evaluation included a CT scan of the whole abdomen which showed an incidental finding of right adrenal mass.
Patient was completely asymptomatic except for an on and off vague right flank pain with no associated fever, unexplained weight loss, tremors, palpitations, or changes in urine. Vital signs were stable and normal. He was not pale and did not have acne, moon facies, buffalo hump, or anterior neck mass. He had regular a cardiac rhythm and the apex beat was not displaced. The abdomen was soft, with no palpable masses, tenderness or violaceous striae. He had no edema, tremors or ecchymosis, and had equal peripheral pulses.

To determine the nature of the mass, biochemical evaluation was done which showed an AM cortisol of $9.91 \mu \mathrm{g} / \mathrm{dl}(7-25 \mu \mathrm{g} / \mathrm{dL})$ and serum aldosterone of $10 \mu \mathrm{g} / \mathrm{dl}(<21 \mathrm{ng} / \mathrm{dL})$. Other biochemical tests including catecholamines were normal and consistent with a nonfunctioning tumor.

Further imaging with MRI of the adrenal glands with contrast revealed a multilobulated mass lesion in the right suprarenal region insinuated between the medial margins of the right lobe of the liver and medial crus of the left hemidiaphragm, measuring about $4.3 \times$ $3.6 \times 7.2 \mathrm{~cm}(\mathrm{AP} \times \mathrm{T} \times \mathrm{Cc})$. (Figure 1) The lesion appeared to be heterogeneous most prominently of signals completely suppressing on the fat saturation sequences, findings consistent with adrenal myelolipoma.

Due to the large size of the tumor and the risk of retroperitoneal bleeding, the patient agreed to an Elective Laparoscopic Adrenalectomy 

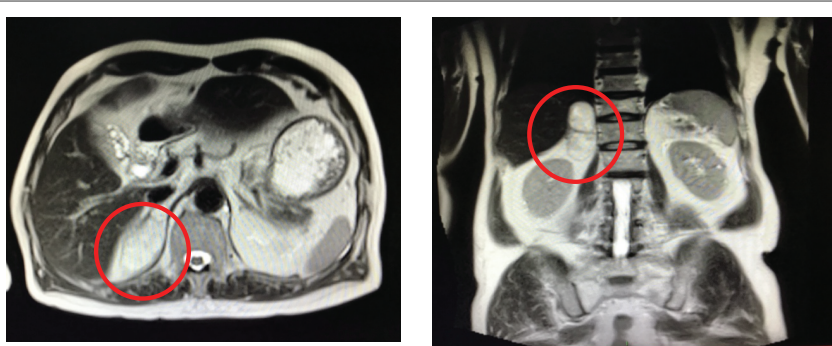

Figure 1: Heterogenous suprarenal mass $(4.3 \times 3.6 \times 7.2 \mathrm{~cm})$ : (right) axial view (left) coronal view.

in our institution. Routine preoperative steroid preparation was done on the morning prior to surgery. No perioperative or postoperative complications were noted. A soft to rubbery, yellow to brown mass measuring $70 \times 65 \times 30 \mathrm{~mm}$ adrenal mass was removed and was then sent for biopsy. Methylprednisolone $20 \mathrm{mg}$ was given immediately post procedure and on the $2^{\text {nd }}$ postoperative day, patient was deemed fit for discharge.

Histopathology report showed a section of a benign neoplasm composed of skeletal muscles and mature adipocytes traversed by delicate fibrous septae consistent with Adrenal Myelolipoma (Figure 2).

\section{Discussion}

Adrenal Myelolipoma is a rare benign neoplasm composed of mature adipose tissue and on occasions a variable amount of haemopoietic elements. It is usually discovered incidentally on autopsy, surgery, or as an incidentaloma on imaging for other reasons. The incidence of Adrenal incidentalomas is now increasing due to increasing use of imaging studies. In the recent American Association of Clinical Endocrinologists (AACE) guidelines on the Management of Adrenal Incidentalomas, it is believed that the incidence is now approaching $8.7 \%$ [5]. A summary of the literature revealed that approximately $80 \%$ of patients with adrenal incidentalomas are nonfunctioning adenoma, 5\% had Subclinical Cushing syndrome (SCS), $5 \%$ had a pheochromocytoma, $1 \%$ had an aldosteronoma, $<5 \%$ had an Adrenocortical Carcinoma (ACC), and 2.5\% had a metastatic lesion; the remaining small percentage of incidentalomas were ganglioneuromas, myelolipomas, or benign cysts [6].

The reported incidence of adrenal myelolipoma varies from $0.08 \%$ to $0.4 \%$, and less than 300 cases are reported in the literature before 2000. A recent review of published cases in 2018 showed that 420 cases have already been reported between 1957 and 2017 on adrenal myelolipoma [7]. The male to female ratio is $1: 1$, and are commonly found in the fifth to seventh decade. Tumor sizes have been reported in literature from a few millimeters to more than $30 \mathrm{~cm}$, but rarely exceed $5 \mathrm{~cm}$. They are mostly unilateral and do not undergo malignant transformation [1]. Several case series have reported the predominance of the tumor in the right adrenal gland, which is yet to be explained [4]. Bilateral tumors occur in about $10 \%$ of cases [1].

Most lesions are small and nonfunctioning and therefore patients are almost always asymptomatic [4]. Occasional cases of adrenal myelolipoma have been reported with Cushing's syndrome, 21-hydroxylase deficiency, and hypenaldostenonism [3,8]. Although they do not represent a hematopoietic source, sometimes they contain precursors of white and red blood cells such as megakaryocytes.
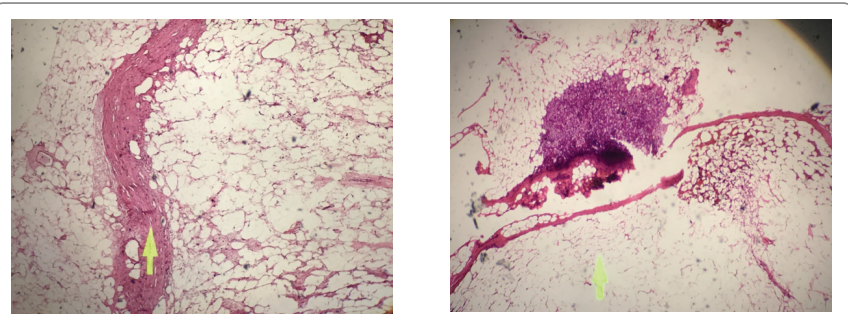

Figure 2: Histopathology finding of the adrenal mass.

One possible etiology of this disease is that the fat component is derived from the mesenchymal stem cells of stromal fat of the adrenal cortex [9]. Subsequently, the circulating hematopoietic cells are recruited possibly due to the release of granulocyte colony stimulating factor by adrenal cortical tissue [9]. However, the most widely accepted theory of its etiology is adrenocortical cell metaplasia in response to stimuli, such as necrosis, inflammation, infection, or stress [10]. This chronic stimulation to the adrenal gland, which is evidenced by the increased incidence of the lesion in the advanced age, apparently triggers the development of benign as well as malignant tumors.

The appearance of myelolipoma on imaging can be diagnostic and is based on the fat content of the lesion. They appear echogenic on ultrasound, and as low attenuation lesions on CT scan. Ultrasound of the abdomen is able to differentiate the supra-renal mass from the kidneys, but it cannot confirm a myelolipoma. As in our case, we have to further do other imaging studies to determine the nature of the suprarenal mass found on ultrasound. On CT scan, adrenal myelolipoma is seen as a hypodense, non-enhancing lesion with attenuation values suggestive of fat. MRI of adrenal myelolipoma characteristically demonstrates a bright signal on T1-weighted and $\mathrm{T} 2$-weighted sequences, consistent with the presence of fat and the lesion enhances brightly after intravenous administration of gadolinium [1]. Decrease in signal with fat suppression or phase cancellation is confirmatory of adrenal myelolipoma [1]. If the diagnosis is in doubt, an image guided needle biopsy could be performed to confirm the diagnosis but this approach bears the risk of rupture and bleeding [8]. Histological examination of a myelolipoma shows adipocytes with interspersed hematopoietic elements, consisting of myeloid and erythroid precursors, as well as, megakaryocytes [4].

The well-recognized complication of adrenal myelolipoma is spontaneous retroperitoneal hemorrhage. No potential of malignancy for adrenal myelolipoma has been reported [4]. The asymptomatic small lesions of less than $4 \mathrm{~cm}$ should be followed up with CT scan or MRI although most experts advocate a simple clinical follow-up without routine radiological investigations.

Our case of adrenal myelolipoma was already $7 \mathrm{~cm}$, which prompted the surgical advice. Surgery is usually indicated in patients with lesion of more than $4 \mathrm{~cm}$ in size due to rare chances of rupture [8]. With the advent of minimally invasive surgery, laparoscopic adrenalectomy has shown a considerable decrease in surgically derived morbidity as well as hospital stay and convalescence [4]. In case of bilateral myelolipoma, a staged tumor removal is preferable, removing the larger one and continuing to observe the contralateral myelolipoma as long as possible in an effort to avoid adrenal insufficiency and a lifetime of steroid replacement [8]. The long-term prognosis of this case is very good and no long-term follow-up is needed [3]. 


\section{Conclusion}

A diagnosis of adrenal myelolipoma is a diagnosis based on routine imaging for other reasons. A suspicious mass in the suprarenal region warrants further testing of this adrenal incidentaloma to determine if the tumor is functioning or not. Radiographic imaging and histology can reach a diagnosis of adrenal myelolipoma. A size more than 4 $\mathrm{cm}$ and the risk of retroperitoneal bleed warrant surgical removal of the tumor using the minimally invasive approach of laparoscopic adrenalectomy to minimize morbidity. Long-term prognosis is very good and warrants no further evaluation post-operatively.

\section{Acknowledgement}

This paper was presented as a poster during the American Association of Clinical Endocrinologists Annual meeting in Boston, USA May 2018.

This paper was partially funded by a research grant of AACE Philippines Research Adocacy.

\section{References}

1. Sudeendra Doddi, Tarun Singhal, Tessa Leake, Prakash Sinha (2009) Management of an incidentally found large adrenal myelolipoma: a case report. Cases J 2: 8414.

2. Kasper DL, Fauci AS, Hauser SL, Longo DL, Jameson JL, et al. (2015) Disorders of the Adrenal Cortex. Harrison's Principles of Internal Medicine. $19^{\text {th }}$ Edition, McGraw Hill Professional, United States 2309-2329.
3. Sandoval MA, Anel-Quimpo J (2010) A giant myelolipoma discovered as an adrenal incidentaloma: radiological, endocrine and pathological evaluation. BMJ Case Rep 2010.

4. Bandar Al Harthi, Muhammad M Riaz, Amal H Al Khalaf, Mohammad Al Zoum, Wafa Al Shakweer (2009) Adrenal myelolipoma a rare benign tumour managed laparoscopically: Report of two cases. J Minim Access Surg 5: 118-120.

5. AACE (2009) Medical Guidelines for the Management of Adrenal Incidentaloma. Endocr Pract 15.

6. Young WF Jr (2000) Management approaches to adrenal incidentalomas. A view from Rochester, Minnesota. Endocrinol Metab Clin North Am 29: 159-185.

7. Decmann Á, Perge P, Tóth M, Igaz P (2018) Adrenal myelolipoma: a comprehensive review. Endocrine 59: 7-15.

8. Cyran KM, Kenney PJ, Mermel DS, Yacoub I (1996) Adrenal myelolipoma. Am J Roentgenol 166: 395-400.

9. Vasanth G Shenoy, Anuroop Thota, Ravi Shankar, Mallikarjun G Desai (2015) Adrenal myelolipoma: Controversies in its management. Indian J Urol 31: 94-101.

10. Nabi J, Rafiq D, Authoy, FN, Sofi GN (2013) Incidental detection of adrenal myelolipoma: a case report and review of literature. Case Rep Urol 2013: 789481. 